\title{
Role of uL3 in Multidrug Resistance in p53-Mutated Lung Cancer Cells
}

\author{
Annapina Russo ${ }^{1, *}$, Assunta Saide ${ }^{1}$, Silvia Smaldone ${ }^{2}$, Raffaella Faraonio ${ }^{3}$ and Giulia Russo ${ }^{1, *}$ \\ 1 Department of Pharmacy, University of Naples "Federico II", Via Domenico Montesano 49, \\ 80131 Naples, Italy; assuntasaide@gmail.com \\ 2 Department of Pharmacology and Systems Therapeutics, Icahn School of Medicine at Mount Sinai, \\ New York, NY 10029, USA; silvia.smaldone@gmail.com \\ 3 Department of Molecular Medicine and Medical Biotechnologies, University of Naples "Federico II", \\ Via Sergio Pansini 5, 80131 Naples, Italy; raffaella.faraonio@unina.it \\ * Correspondence: annapina.russo@unina.it (A.R.); giulia.russo@unina.it (G.R.); \\ Tel.: +39-081-678-414 (A.R.); +39-081-678-415 (G.R.)
}

Academic Editor: Li Yang

Received: 18 January 2017; Accepted: 1 March 2017; Published: 3 March 2017

\begin{abstract}
Cancer is one of the most common causes of death among adults. Chemotherapy is crucial in determining patient survival and quality of life. However, the development of multidrug resistance (MDR) continues to pose a significant challenge in the management of cancer. In this study, we analyzed the role of human ribosomal protein uL3 (formerly rpL3) in multidrug resistance. Our studies revealed that uL3 is a key determinant of multidrug resistance in $p 53$-mutated lung cancer cells by controlling the cell redox status. We established and characterized a multidrug resistant Calu-6 cell line. We found that uL3 down-regulation correlates positively with multidrug resistance. Restoration of the uL3 protein level re-sensitized the resistant cells to the drug by regulating the reactive oxygen species (ROS) levels, glutathione content, glutamate release, and cystine uptake. Chromatin immunoprecipitation experiments and luciferase assays demonstrated that uL3 coordinated the expression of stress-response genes acting as transcriptional repressors of solute carrier family 7 member $11(x C T)$ and glutathione S-transferase $\alpha 1$ (GST- $\alpha 1)$, independently of Nuclear factor erythroid 2-related factor 2 (Nrf2). Altogether our results describe a new function of $\mathrm{uL} 3$ as a regulator of oxidative stress response genes and advance our understanding of the molecular mechanisms underlying multidrug resistance in cancers.
\end{abstract}

Keywords: ribosomal protein; nucleolar stress; uL3; multidrug resistance; lung cancer; Nrf2; $x C T$; GST- $\alpha 1$; MDR1; chemoresistance

\section{Introduction}

Lung cancer is one of the most common causes of cancer-related death among adults [1]. Chemotherapy is recognized as an important component of treatment for all stages of lung cancer and is crucial in determining patient survival and quality of life [2]. However, intrinsic or acquired multidrug resistance (MDR) is the main reason for tumor recurrence [3]. Therefore, it is crucial to understand the molecular basis underlying the mechanisms of MDR. Here, we used 5-Fluorouracil (5-FU) to establish a multidrug resistant lung cell model [4,5].

In addition to its effect on thymidylate synthase inhibition and DNA synthesis, 5-FU may also influence RNA metabolism [6]. It is known that the nucleolus is a ribosome-supplying organelle, however a growing body of evidence suggests that the nucleolus plays intriguing novel roles in sensing cellular stress signals [7]. Under stress conditions, the structure of the nucleolus is perturbed and some nucleolar proteins, including ribosomal proteins ( $\mathrm{rp}$ ), are released from the nucleolus to the 
nucleoplasm, where they associate with Mouse Double Minute 2 (MDM2) to inhibit its activity and stabilize p53 [7]. In yeast, it has been reported that the nucleolus can function as an oxidative stress sensor [8].

It has been demonstrated that some rp play a key role in cell resistance to chemotherapeutic drugs [9]. Specifically, upon 5-FU induced nucleolar stress, a subset of rp including uL18 (rpL5), uL5 (rpL11), and uL14 (rpL23) are released from the ribosome to activate p53 through MDM2 inhibition [10]. Recently, our studies focused on post-transcriptional regulation [11-14] and extra-ribosomal functions of ribosomal proteins [11,15]. In particular, we demonstrated that uL3 (formerly rpL3) [16] is involved in the regulation of its own expression by regulating the splicing of its own transcript $[17,18]$. In addition, we identified a new molecular pathway activated in the cell response to 5-FU that is p53-independent but still rp-dependent. We demonstrated that upon ribosomal stress induced by 5-FU, ribosome free uL3 becomes a regulator of p21 [19,20], cystathionine- $\beta$-synthase (CBS) [21-23], and NFKB [22] expression and mediates apoptosis through the activation of the mitochondrial apoptotic response pathway. Of note, our data indicated that uL3 status is associated to chemoresistance since the loss of uL3 makes chemotherapeutic drugs, such as 5-FU, oxaliplatin (L-OHP), Actinomycin D, and Cisplatin ineffective in colon and lung cancer cells lacking active p53 [21-24]. According to this, we reported that the resistance of A549 lung cancer cells to Cisplatin correlates to the loss of $\mathrm{uL3}$ expression [25]. Evidence of the role of some rp mediating drug resistance has been previously reported. To date, eL36 (rpL36) plays a role in cisplatin resistance in human carcinoma cells [26], the radiosensitivity in NSCLC (non-small cell lung cancer) cells is regulated by uS3 (rpS3) [27], and eL24 (rpL24) may have effects on drug resistance mechanisms in hepatocellular carcinoma HepG2 cells [28].

In the present study, we identified uL3 (formerly rpL3) as a key molecule conferring multidrug resistance to lung cancer cells lacking p53 and elucidated the molecular mechanism involved in this process. We have established and characterized a $p 53$-mutated lung cancer cell line resistant to 5-FU. We have demonstrated that in these cells, uL3 expression is down-regulated upon acquired multidrug resistance but its restoration re-sensitized the cells to 5-FU through the regulation of reactive oxygen species (ROS) levels, glutathione (GSH) content, glutamate release, and cystine uptake. The molecular mechanism by which this occurs involves uL3-mediated control of the stress-response gene expression. Specifically, uL3 functions as a transcriptional regulator of solute carrier family 7 member $11(x C T)$ and glutathione S-transferase $\alpha 1$ (GST- $\alpha 1)$, independently of Nuclear factor erythroid 2-related factor 2 (Nrf2).

These data imply that there is a mechanistic link between the response pathway to drug-induced ribosomal stress and the development of drug resistance and suggest the possibility of targeting uL3 to modulate the redox status of cancer cells for future therapeutic purposes in order to overcome MDR.

\section{Results}

\subsection{Production of the MDR Resistant p53-Mutated Lung Cancer Cell Line}

To explore the mechanisms of MDR, we established a multidrug resistant Calu- 6 subline (rCalu-6 cells) derived from the parental Calu- 6 sensitive cells. To this aim, Calu- 6 cells were continuously selected with an increasing concentration of 5-FU (from 2.5 to $25 \mu \mathrm{M}$ ) over a period of about 10 months in order to generate the corresponding age and passage-matched 5-FU resistant cell line designated rCalu-6. Maintenance of the resistant subline was continued at $20 \mu \mathrm{M}$. Figure 1 shows the cell survival curves of 5-FU sensitive and 5-FU resistant cells, and Calu- 6 and rCalu- 6 cells, respectively, after treatment with different concentrations of 5-FU for $48 \mathrm{~h}$. Interestingly, the drug-adapted rCalu- 6 cell line showed a significantly higher $\mathrm{IC}_{50}$ compared with parental Calu- 6 cells $(52.3 \pm 0.02$ vs. $4.5 \pm 0.08$, respectively). The degree of resistance to 5 -FU was evaluated as the ratio of the $\mathrm{IC}_{50}$ values of 5 -FU resistant Calu- 6 cells to that of parental cells (Table 1). The results shown in Figure 1A and Table 1 indicate that the Calu- 6 subline was successfully established as a 5-FU-resistant lung cancer cell line with a degree of resistance to 5-FU 11.7-fold higher than parental Calu-6 cells. 


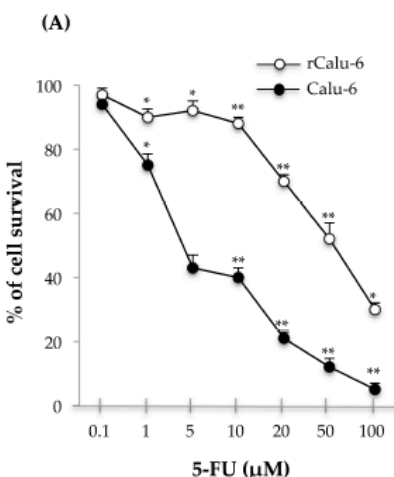

(C)

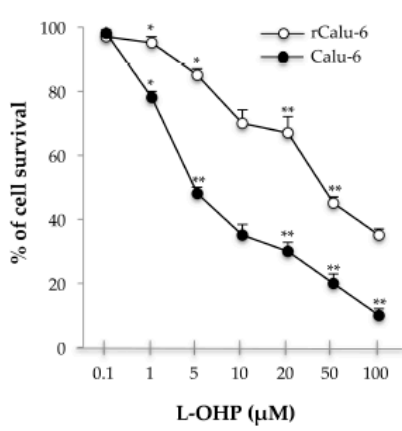

(B)

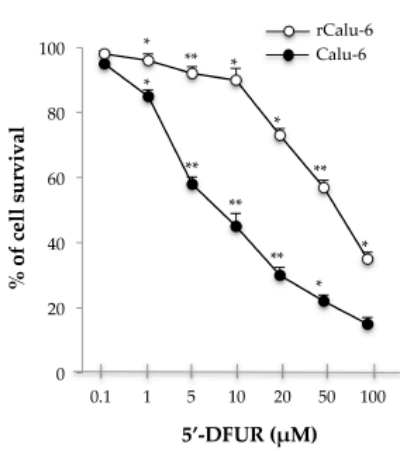

(D)

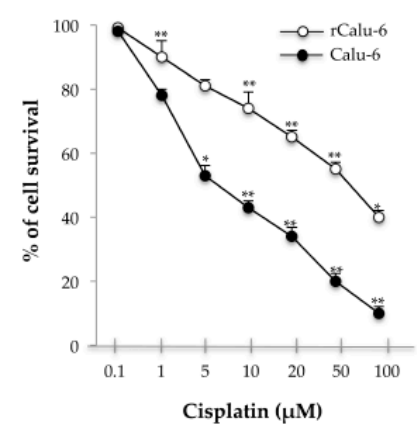

Figure 1. In vitro sensitivity of Calu-6 and rCalu-6 cells to 5-Fluorouracil (5-FU), 5'-Deoxy-5fluorouridine (5'-DFUR), Oxaliplatin (L-OHP), and Cisplatin. Cells were cultured with increasing concentrations of 5-FU for $48 \mathrm{~h}$ and cell growth was assessed by sulforhodamine B (SRB) colorimetric assay. Cell growth was expressed as the percentage of control for each time point. ${ }^{* *} p<0.01,{ }^{*} p<0.05$ vs. untreated cells. The results illustrated in Figures 1-5 are representative of three independently performed experiments; error bars represent the standard deviation.

Table 1. Antiproliferative effect of anticancer drugs on Calu- 6 and rCalu- 6 cells. The tesistance index (RI) is reported as $\mathrm{IC}_{50}$ rCalu-6/ $\mathrm{IC}_{50}$ Calu-6.

\begin{tabular}{cccc}
\hline \multirow{2}{*}{ Drug } & $\mathbf{I C}_{\mathbf{5 0}}(\boldsymbol{\mu M})$ & r/Calu-6 & $\boldsymbol{p}$-Value (Calu-6 vs. rCalu-6) \\
\cline { 2 - 2 } & Calu-6 & & \\
\hline 5-FU & $4.5 \pm 0.08$ & $52.3 \pm 0.02(\mathrm{RI}=11.7 \mathrm{X})$ & $<0.01$ \\
$5^{\prime}$-DFUR & $8.35 \pm 0.06$ & $56.7 \pm 0.03(\mathrm{RI}=7 \mathrm{X})$ & $<0.05$ \\
L-OHP & $5 \pm 0.07$ & $50 \pm 0.03(\mathrm{RI}=10 \mathrm{X})$ & $<0.01$ \\
Cisplatin & $10 \pm 0.02$ & $60 \pm 0.05(\mathrm{RI}=6 \mathrm{X})$ & $<0.01$ \\
\hline
\end{tabular}

\subsection{Cross-Resistance Profiles of rCalu-6 Cells}

Since drug resistance involves the resistance to one drug accompanied by the resistance to several other anticancer drugs [29], we evaluated whether rCalu-6 cells acquired cross-resistance to other conventional anticancer drugs such as 5'-deoxy-5-fluorouridine (5'-DFUR) (Figure 1B), oxaliplatin (L-OHP, Figure 1C) and cisplatin (Figure 1D). Interestingly, cross-resistance to other classes of anti-cancer agents was observed, indicating potential multi-resistant phenotypes. The degree of resistance of rCalu-6 cells to 5'-DFUR, cisplatin, and L-OHP were 7, 6, and 10-fold higher than that of parental sensitive Calu-6 cells, respectively (Table 1). These results indicate that rCalu- 6 cells show resistance to multiple drugs, representing an interesting tool to study MDR in lung cancer. 


\subsection{Expression Analysis of Genes Related to Drug Resistance in rCalu-6 Cells}

According to previous studies, 5-FU resistance is caused by an increase in the 5-FU-degrading enzyme dihydropyrimidine dehygrogenase (DPYD) and 5-FU-targeting enzyme thymidylate synthase (TS) [30]. On the other hand, ABC family proteins, such as Multi Drug Reactivity 1 (MDR1), are related to multiple drug resistance in cancer. We have previously demonstrated that uL3 is able to negatively control Pgp expression in colon cancer cells by regulation of MDR1 mRNA stability [24]. Moreover, we also demonstrated that the loss of uL3 and the consequent upregulation of the cystathionine $\beta$ synthase (CBS) enzyme correlated with ineffectiveness of 5-FU in lung and colon cancer cell lines [23]. Based on these data, we employed quantitative real-time PCR (qRT-PCR) to assess the expression profile of TS, DPYD, MDR1, uL3, and CBS in Calu-6 cells and rCalu-6 cells. As shown in Figure 2A, the resistant cell line showed an increase in MDR1 and CBS mRNA amounts of about 2-fold compared to those observed in 5-FU sensitive Calu-6 cells. In contrast, for the condition of 5-FU resistance, the mRNA levels of $u L 3$ were strongly decreased in the resistant cell line compared to control cells.

(A)

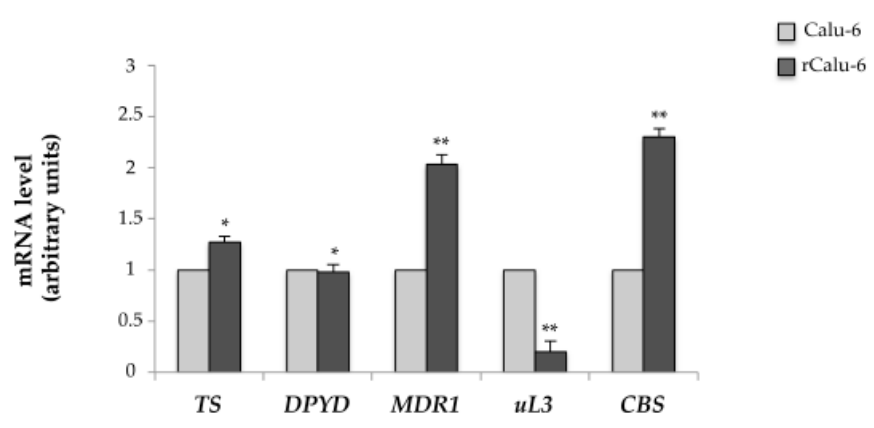

(B)
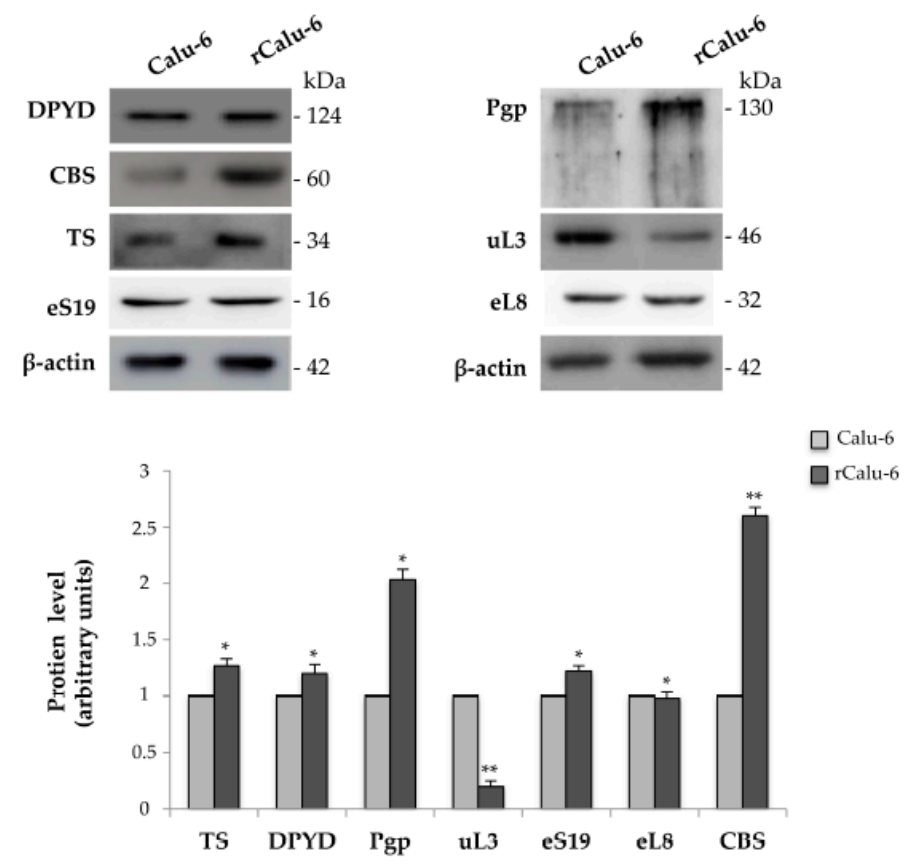

Figure 2. Analysis of mRNAs and proteins related to chemoresistance. (A) Total RNA from Calu-6 and rCalu- 6 cells was subjected to Reverse Transcription quantitative Polymerase Chain Reaction (RT-qPCR) with primers specific for the indicated mRNAs. The quantification of signals is shown. ${ }^{* *} p<0.01$, * $p<0.05$ vs. mRNA levels in Calu 6 cells set at 1 ; (B) Protein extracts from Calu- 6 and rCalu- 6 cells were analyzed by Western blotting with antibodies against the indicated proteins. $\beta$-actin was used as the loading control. The quantification of signals is shown. ${ }^{* *} p<0.01,{ }^{*} p<0.05$ vs. protein levels in Calu 6 cells set at 1 . 
The mRNA expression of TS and DPYD did not differ between drug resistant and sensitive cell lines. The expression of these genes at the protein level by Western blot analysis in Calu- 6 and rCalu- 6 cells was consistent with the mRNA analysis (Figure 2B). The expression levels of eS19 and eL8, two arbitrary proteins of large and small subunits, respectively, remained unchanged.

\section{4. uL3 Mediates Anti-Oxidative Cell Response in rCalu-6 Cells}

It is known that the toxicity of antitumor drugs may largely depend on the redox status of the cells. The observed decreased expression of uL3 in rCalu- 6 led us to hypothesize that the levels of uL3 would be functionally related to ROS production in these cells. To test this hypothesis, we first examined ROS production in Calu- 6 cells and the resistant parental subline. To this aim, Calu- 6 and rCalu- 6 cells, were treated with $10 \mu \mathrm{M}$ 5-FU for $48 \mathrm{~h}$ and then the ROS content was determined. As expected, we found that 5-FU treatment increased ROS production in 5-FU sensitive Calu-6 cells compared to the untreated cells, while in the resistant rCalu- 6 cell line and uL3 $\Delta$ Calu- 6 cells, in which uL3 expression was stably switched off, 5-FU treatment failed to induce ROS production (Figure 3A). Next, we monitored the levels of intracellular GSH, that is known to play an important role in providing protection against oxidative damage in the same cells. As shown in Figure 3B, the GSH content in rCalu- 6 and uL3 $\Delta$ Calu- 6 treated cells was improved compared with that found in the untreated cells. As expected, in treated Calu- 6 cells the level of GSH was significantly lower than in the untreated cells. Next, since cystine is essential for the generation of GSH, we tested cystine uptake and the release of glutamate in the same cells. Figure 3C,D shows that cystine uptake and glutamate release were strongly inhibited in Calu- 6 cells after drug treatment. On the contrary, the acquisition of drug resistance was associated to a significant increase of cystine uptake and glutamate release after 5-FU treatment. These data clearly suggest that oxidative stress target genes are involved in the molecular mechanism for acquiring MDR resistance in Calu- 6 cells. Interestingly, we demonstrated that the observed alteration in the cell redox status of resistant cells was specifically mediated by uL3. In fact, the enforced expression of uL3 in rCalu-6/uL3 was associated to the loss of chemoresistance as observed by the inversion of the redox status in these cells (Figure 3A-D). Additionally, we performed cell proliferation assays to evaluate the cell response to 5-FU upon alteration of uL3 status in the cells. As shown in Figure 3E, the down-regulation of uL3 (rCalu- 6 cells and uL3 $\Delta$ Calu-6) was associated to a marked reduced cell response to 5-FU. The restoration of $\mathrm{uL3}$ (rCalu-6/uL3) re-sensitized rCalu-6 cells to 5-FU causing a decrease of the percent of cell survival after 5-FU treatment. Interestingly, the over-expression of eL8 in rCalu-6 cells failed to overcome the chemoresistance and in Calu-6 cells did not affect the cell response to $5-\mathrm{FU}$, demonstrating the specificity of $\mathrm{uL} 3$ in these processes. The central role of $\mathrm{uL} 3$ in mediating the cell response to 5-FU was also confirmed by the results from the clonogenic survival assays (Figure 3E).

All together, these results demonstrated that $\mathrm{uL} 3$ is essential to mediate the cell response to 5-FU in Calu-6 cells by control of the cell redox status. 

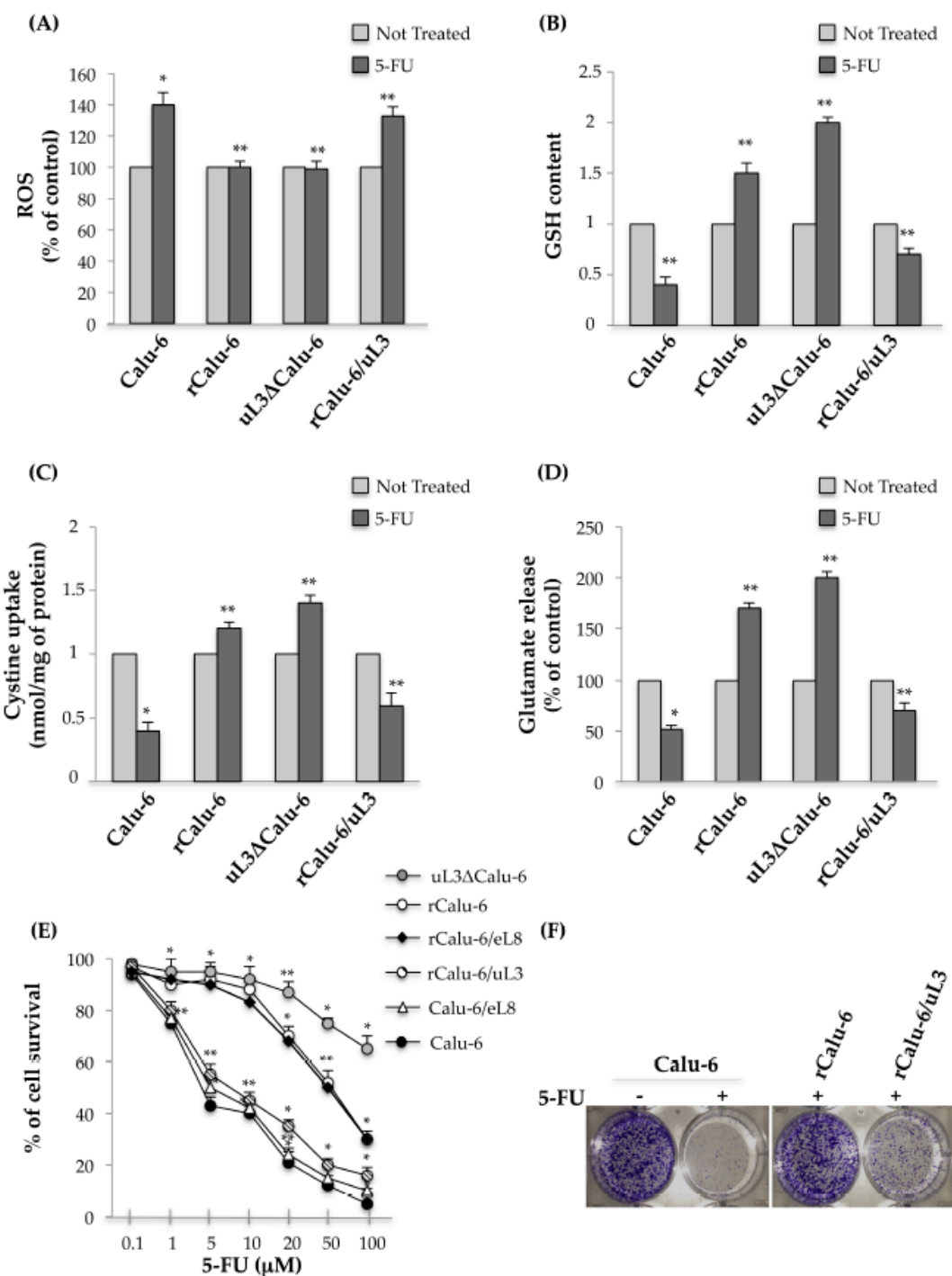

(F)

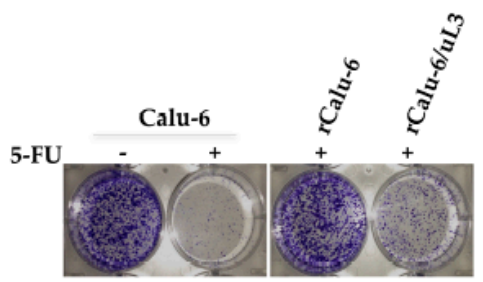

Figure 3. uL3 contributes to Reactive Oxygen Species (ROS) defense in rCalu-6 cells. (A) Histogram quantifying ROS production in indicated cell lines after treatment with 5-FU. ${ }^{* *} p<0.01,{ }^{*} p<0.05$ vs. respective untreated cells set at 1; (B) Glutathione (GSH) content in Calu- 6 cells and derivative sublines after treatment with 5-FU. The quantification of signals is shown. ${ }^{* *} p<0.01,{ }^{*} p<0.05$ vs. respective untreated cells set at 1 ; (C) Changes in cystine uptake after incubation of indicated cells with 5-FU. ${ }^{* *} p<0.01,{ }^{*} p<0.05$ vs. respective untreated cells set at 1 ; (D) Evaluation of glutamate release in indicated cell lines after treatment with 5-FU. ${ }^{* *} p<0.01,{ }^{*} p<0.05$ vs. respective untreated cells set at 1 ; (E) uL3 $\Delta$ Calu-6, rCalu-6, rCalu-6/uL3, rCalu-6/eL8, Calu-6/eL8 and Calu-6 cells were cultured with increasing concentrations of 5-FU for $48 \mathrm{~h}$ and cell growth was assessed by SRB colorimetric assay. Cell growth was expressed as the percentage of control for each time point. Ectopic expression of uL3 (rCalu-6/uL3 cells) abolished resistance to 5-FU. ${ }^{* *} p<0.01,{ }^{*} p<0.05$ vs. Calu 6 cells; (F) Representative image of the clonogenic analysis for cell proliferation of Calu-6, rCalu-6, and rCalu-6/uL3 after treatment with $10 \mu \mathrm{M} 5-\mathrm{FU}$ for $48 \mathrm{~h}$.

\section{5. uL3 Reduces $x$ CT and GST- $\alpha 1$ Expression Levels}

To better characterize the molecular mechanism by which uL3 controls the alteration in cell-redox status in MDR resistance, we firstly assessed the intracellular levels of Nrf2 in drug sensitive and resistant Calu-6 cells, a crucial transcriptional regulator that activates various genes involved in oxidative stress, together with $x$ CT and GST- $\alpha 1$, as representative Nrf2-regulated antioxidant defense/drug metabolism genes. To this aim, proteins extracted from Calu- 6 treated or not treated 
with $10 \mu \mathrm{M}$ 5-FU for $48 \mathrm{~h}$, uL3 $\Delta$ Calu-6, and rCalu- 6 cells were analyzed by Western blotting with antibodies against Nrf2, xCT, and GST-A1. Figure 4A shows that in Calu- 6 cells, Nrf2 levels are not significantly modified by 5-FU treatment while xCT and GST-A1 expression levels were markedly decreased. Interestingly, in uL3 $\Delta$ Calu- 6 and rCalu- 6 cells, in which uL3 was respectively stably silenced and significantly down-regulated, we observed a marked increase of the XCT and GST-A1 levels. On the contrary, Nrf2 levels were not significantly altered in both cell lines and immunoprecipitation experiments demonstrated the absence of an interaction between Nrf2 and uL3 (Figure S1).

(A)
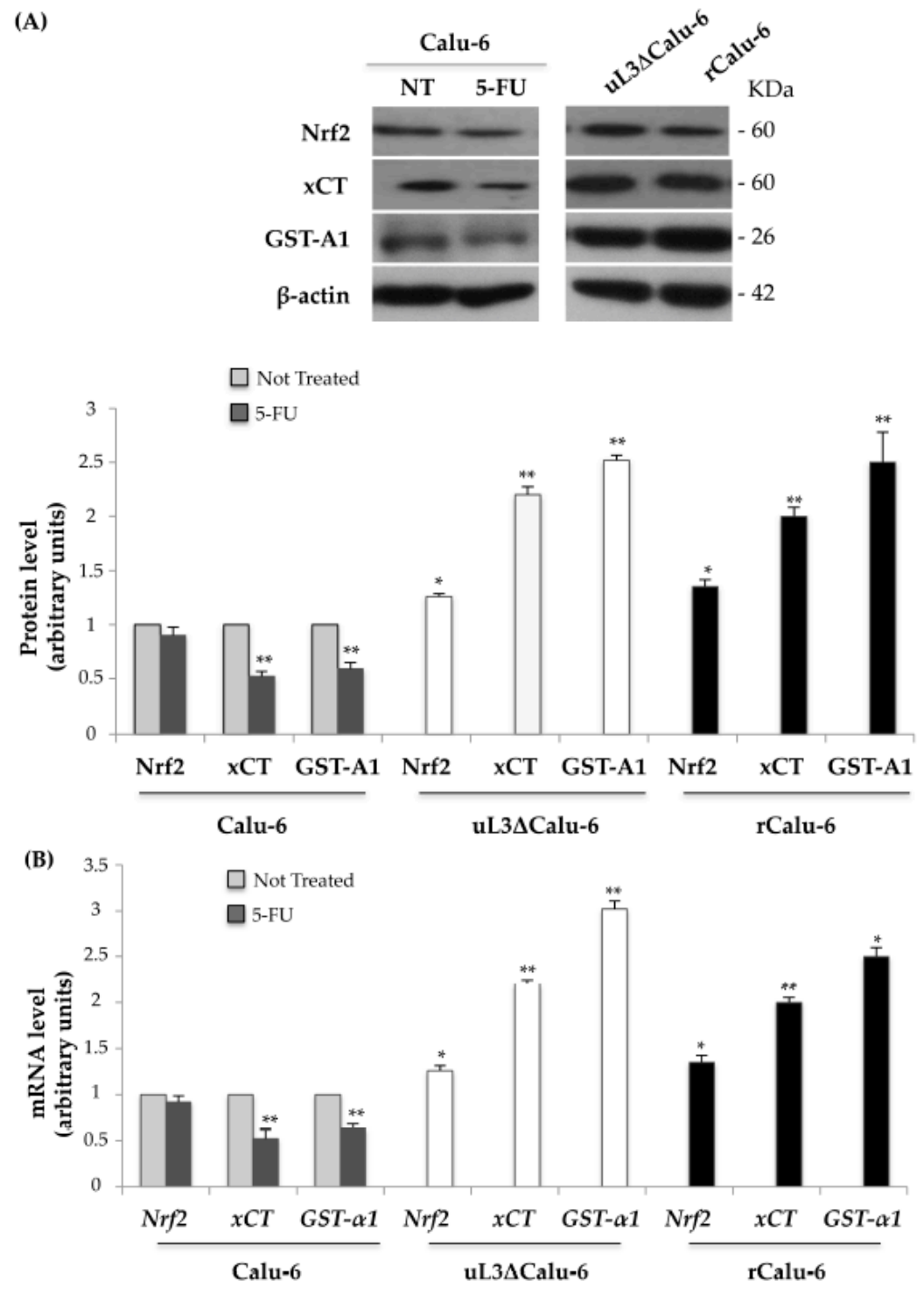

Figure 4. Influence of uL3 on Nrf2, xCT, and GST-A1 expression levels in Calu-6 cells and resistant sublines. (A) Protein extracts from Calu- 6 cells treated or not with $10 \mu \mathrm{M} 5$-FU for $48 \mathrm{~h}$, uL $3 \Delta$ Calu-6 and rCalu- 6 cells were analysed by Western blotting with the indicated antibodies. Anti- $\beta$-actin was used as the loading control. The quantification of signals is shown. ${ }^{* *} p<0.01,{ }^{*} p<0.05$ vs. protein levels in untreated Calu- 6 cells set at 1; (B) Total RNA from Calu-6 treated or not with $10 \mu \mathrm{M}$ 5-FU for $48 \mathrm{~h}, \mathrm{uL} 3 \Delta$ Calu- 6 and rCalu- 6 cells were subjected to PPCR with primers specific for the indicated genes. The quantification of signals is shown. ${ }^{* *} p<0.01,{ }^{*} p<0.05$ vs. mRNA levels in untreated Calu-6 cells set at 1 . 
These results clearly demonstrate a central role of uL3 in the regulation of $x C T$ and GST- $\alpha 1$ expression in response to 5-FU treatment and in multidrug resistance.

Next, we analyzed the expression of $x C T$ and GST- $\alpha 1$ at the mRNA level. To this aim, the total RNA from Calu-6, treated or not treated with $10 \mu \mathrm{M}$ 5-FU for $48 \mathrm{~h}$, uL3 $\Delta$ Calu-6, and rCalu-6 cells was isolated and analyzed by quantitative RT-PCR using primers specific for Nrf2, $x C T$, and GST- $\alpha 1$ mRNAs (Figure 4B). Consistent with the protein analysis, the treatment of Calu- 6 cells with 5-FU resulted in a small reduction of the Nrf2 mRNA level while $x C T$ and GST- $\alpha 1$ mRNA levels were significantly decreased. Interestingly, in uL $3 \Delta$ Calu- 6 and rCalu- 6 cells the absence or the reduced expression of uL3, respectively, was associated to an increase in the $x C T$ and GST- $\alpha 1$ mRNA levels. The analysis of $x C T$ and GST- $\alpha 1$ mRNA levels in rCalu- 6 over-expressing uL3 (rCalu-6/uL3) was consistent with these data (Figure S2A). In addition, we evaluated the influence of 5-FU on $x C T$ and GST- $\alpha 1$ mRNA levels in Calu-6, $\Delta \mathrm{uL} 3 /$ Calu-6, and rCalu-6 cells. As expected, in the $\Delta \mathrm{uL} 3 / \mathrm{Calu}-6$ and rCalu- 6 cells, the levels of $x C T$ and GST- $\alpha 1$ were not decreased after 5-FU treatment, in contrast to Calu-6 cell line (Figure S2B).

Altogether these results indicate that uL3 was necessary for regulating $x C T$ and GST- $\alpha 1$ expression in response to 5-FU treatment and in drug resistance.

\section{6. uL3 Is a Transcriptional Repressor of $x$ CT and GST- $\alpha 1$}

Recently, we have demonstrated that after drug treatment the ribosome-free uL3 behaves as a transcription factor [22], thus we became interested in determining if the uL3-mediated down-regulation of $x C T$ and GST- $\alpha 1$ expression occurred via the inhibition of gene transcription. To test the presence of uL3 on $x C T$ and GST- $\alpha 1$ gene promoters, Calu- 6 cells untreated or treated with $10 \mu \mathrm{M}$ 5-FU for $48 \mathrm{~h}$, were collected and subjected to Chromatin immunoprecipitation experiments by using anti-uL3 antibodies and anti-IgG as negative control.

The presence of uL3 in DNA-immunoprecipitated complexes was assayed by Western blotting (Figure 5A). qPCR assays on the samples were performed as previously reported [19]. Figure 5A shows that $\mathrm{uL} 3$ was able to bind to $x C T$ and GST- $\alpha 1$ promoters. Interestingly, after 5-FU treatment, this binding was significantly increased compared to that observed in the control, untreated Calu- 6 cells.

Next, to test the role of uL3 on $x C T$ and GST- $\alpha 1$ promoter activities in the response to drug exposure and in the condition of acquired MDR resistance, we performed reporter luciferase assays. To this aim, Calu- 6 cells untreated or treated with $10 \mu \mathrm{M} 5-\mathrm{FU}$, rCalu-6, and uL3 $\Delta$ Calu- 6 cells were transiently transfected with the $x C T$ (Figure 5B) or GST- $\alpha 1$ (Figure 5C) promoter luciferase reporter constructs. Figure $5 \mathrm{~B}, \mathrm{C}$ shows that 5 -FU treatment significantly reduced $x C T$ and GST- $\alpha 1$ promoter activity in Calu- 6 cells. On the contrary, the transcriptional activity of these promoters was greatly increased in the condition of uL3 silencing (uL3 $\Delta$ Calu- 6 cells) and in rCalu- 6 cells where uL3 expression was down-regulated. To demonstrate that the observed up-regulation of $x C T$ and GST- $\alpha 1$ promoter activity in rCalu- 6 and uL3 3 Calu- 6 cells was specifically mediated by uL3 and independent from Nrf2, we performed analogous experiments in the conditions of Nrf2 silencing or uL3 overexpression. As shown in Figure 5B,C, Nrf2 depletion did not cause any alteration on $x C T$ or GST- $\alpha 1$ promoter activities. Ectopic expression of uL3 dramatically reduced $x C T$ and GST- $\alpha 1$ promoter trans-activation. These results strongly indicate that $\mathrm{UL} 3$ is an important player in the transcriptional regulation of $x C T$ and GST- $\alpha 1$ genes, acting as a negative regulator of their expression independently of Nrf2. 

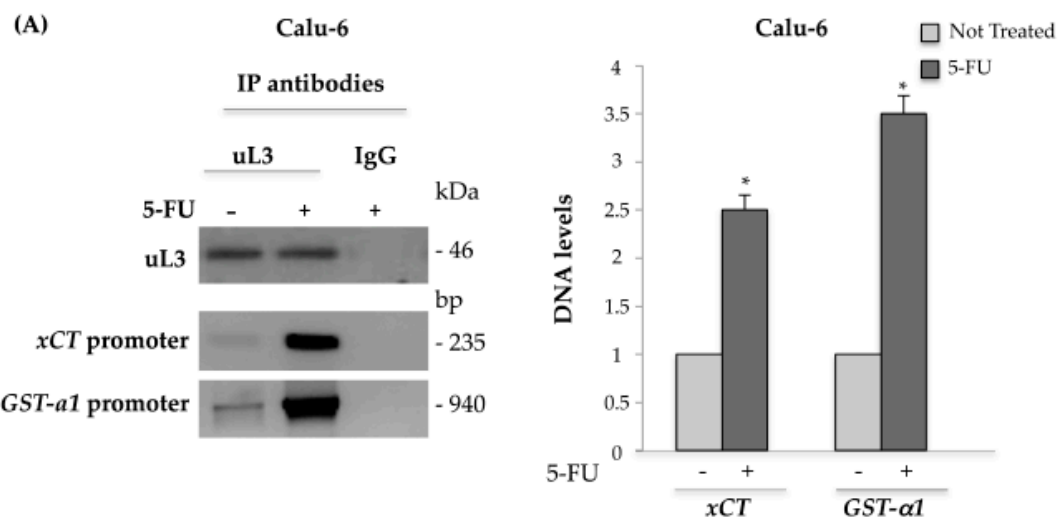

(B)

(C)
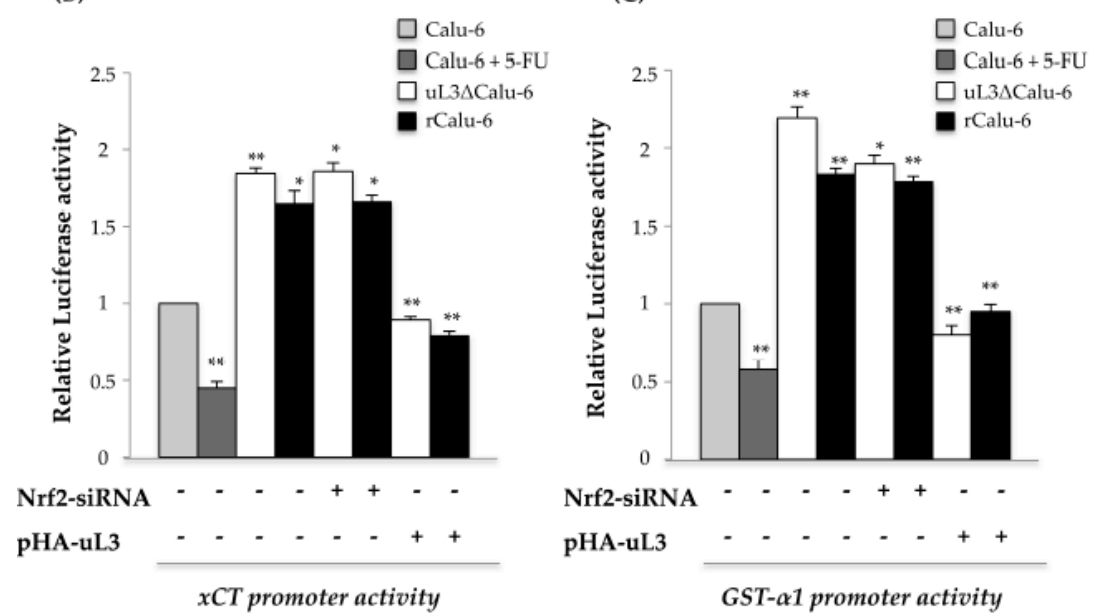

Figure 5. Analysis of the interaction between $\mathrm{uL} 3$ and $x C T$ and GST- $\alpha 1$ gene promoters. (A) Protein samples of DNA-uL3 or DNA-IgG immunocomplexes from Calu-6 cells untreated or treated with $10 \mu \mathrm{M} 5$-FU for $48 \mathrm{~h}$ were analysed by Western blotting with antibodies against uL3. Note the absence of signal in the DNA-IgG immunocomplex. The same DNA-immunoprecipitates were subjected to qPCR with primers specific for $x$ CT or GST- $\alpha 1$ gene promoters. The quantification of signals is shown. $* p<0.05$ vs. DNA levels in untreated cells set at 1 . Calu- 6 cells treated or not treated with $10 \mu \mathrm{M} 5$-FU for $48 \mathrm{~h}, \mathrm{uL} 3 \Delta$ Calu- 6 and rCalu- 6 cells were transiently transfected with (B) $x C T$ promoter luciferase reporter plasmid or (C) GST- $\alpha 1$ promoter luciferase reporter plasmid in presence or absence of Nrf2 siRNA or pHA-uL3 plasmid. Analysis of the relative luciferase activity, normalized against Renilla Luciferase (pRL) activity, of the samples is shown. ${ }^{* *} p<0.01,{ }^{*} p<0.05$ vs. untreated Calu- 6 cells set at 1.

\section{Discussion}

In this study, we used human Calu-6 cells as a model system to establish a p53-mutated multidrug resistant lung cancer cell line. By the stepwise increase of the 5-FU concentration in the growth medium and selection of drug-resistance colonies for ten months, we successfully established a 5-FU resistant cell line, rCalu-6, with a resistance index of 11.7. In addition, rCalu- 6 cells acquired cross-resistance to L-OHP and cisplatin. To the best of our knowledge, this is the first study on the production of a multidrug resistant Calu- 6 cell line. This should be useful to study the mechanisms underlying drug resistance of $p 53$ mutated lung cancer.

The determinants of the resistance mechanisms to 5-FU include the enzymes involved in the 5-FU metabolism pathway [30]. To exert its cytotoxic effects, 5-FU is converted to its active metabolites by several enzymes and the levels of these enzymes have been associated with 5-FU sensitivity [6]. 
Previous results reported that the enhanced expression of dihydropyrimidine dehygrogenase (DPYD) and thymidylate synthase (TS), a 5-FU-degrading enzyme and a 5-FU-targeting enzyme, respectively, correlated with the development of 5-FU resistance [30]. However, we found that the expression of $D P Y D$ and TS was not enhanced in rCalu-6 cells. This suggests that the mechanisms of 5-FU resistance of rCalu- 6 cells differ from those generally reported. Interestingly, the acquired drug resistance was associated to the modification of uL3 expression resulting in a decrease of its intracellular amount. We have also previously observed a reduction of uL3 levels in Cisplatin resistant A549 cells [25] that, unlike Calu- 6 cells, are proficient for p53, suggesting that the loss of uL3 could be considered a general mechanism of multidrug resistance independent of p53 status. Furthermore, we demonstrated that the loss of uL3 was associated with the up-regulation of the MDR1 mRNA level and Pgp transporter encoded by MDR1 in colon cancer cells [24]. According to this, the multidrug resistance in rCalu-6 cells is associated to the high expression of the human MDR1 gene and the Pgp protein levels.

It is known that the toxicity of antitumor drugs may largely depend on the intracellular level of reduced GSH that represents the first line antioxidative defense mechanism [31]. Increased resistance to cancer chemotherapeutic agents is often associated to elevated levels of GSH due to the protective conjugation and detoxification effects of GSH. To date, it has been demonstrated that the increase of GSH concentration or GST expression are linked to chemoresistance pathways in human breast cancer cells [32]. Pathways involved in GSH production and regeneration are controlled by Nrf2, a crucial regulator of the expression of different genes involved in oxidative stress response and drug resistance [33-35]. In order to evaluate the role of the GSH/GST system in rCalu-6 cells, we monitored the levels of intracellular GSH in these cells and in its parent cell line. rCalu- 6 cells were shown to have higher GSH levels than the sensitive Calu- 6 cells after 5-FU treatment. These effects are possibly mediated through up-regulated expression of $x \mathrm{CT}$, causing an increase of the GSH level and inducing the antioxidative defense. $\mathrm{xCT}$ and Cystathionine gamma-lyase (CSE) are two important providers of intracellular cysteine, the precursor for the generation of GSH. xCT is the catalytic light chain of system $\mathrm{xc}^{-}$cystine/glutamate antiporter, an $\mathrm{Na}^{+}$-independent heterodimeric amino-acid transport system that functions as the exchange system for the uptake of extracellular cystine in exchange for the release of intracellular glutamate, at a 1:1 ratio [36]. Once inside the cells, cystine is rapidly reduced to cysteine, the rate-limiting substrate for GSH synthesis involved in many physiological processes [37]. Our data showed that in rCalu- 6 cells the cystine uptake and glutamate release were strongly increased, suggesting that oxidative stress target genes are involved in the molecular mechanism for acquiring multidrug resistance. We have previously demonstrated that uL3 potentiates the cytotoxicity of 5-FU [20-23] and we have also studied the effect of the 5-FU/uL3 combination by using specific delivery systems [38].

Of note, when uL3 was restored, rCalu-6 cells were resensitized to 5-FU, revealing that the restoration of uL3 could be a strategy for reversing 5-FU resistance and to resensitize 5-FU resistant tumors lacking p53 and uL3. Analysis of $x C T$ and GST- $\alpha 1$ mRNA levels reveales that the transcription of these genes is strongly up-regulated upon multidrug acquired resistance. In rCalu- 6 cells, the down-regulation of uL3 is associated to an increase in $x C T$ and GST- $\alpha 1$ gene promoter activity. These two genes are known targets of Nrf2. The unchanged expression of Nrf2 in rCalu- 6 and the absence of an interaction between Nrf2 and uL3 (Figure S1) suggest an Nrf2-independent regulation of $x C T$ and GST- $\alpha 1$ gene expression in our experimental conditions. Accordingly, Nrf2 silencing in rCalu- 6 cells did not cause any alteration on $x C T$ and GST- $\alpha 1$ promoter activity compared to cells expressing Nrf2. All together, these results indicate that uL3 controls the transcription of these genes independently of Nrf2. Although it is well established that cystine and glutamate are substrates of system $\mathrm{xc}^{-}$, it has been recently demonstrated that cystathionine is a novel substrate of the cystine/glutamate transporter [39]. Cystathionine- $\beta$-synthase (CBS), a key enzyme in the trans-sulfuration metabolic pathway involved in the modulation of $\mathrm{H}_{2} \mathrm{~S}$ production [40,41], converts homocysteine to cystathionine, which is converted by CSE to cysteine required for synthesis of the antioxidant glutathione GSH [37]. Silencing CBS severely reduces cellular GSH levels [42]. We have 
previously demonstrated that $\mathrm{uL} 3$ is a negative regulator of CBS expression both at the transcriptional and post-translational levels [23]. rCalu-6 cells express low level of uL3 and high level of CBS. It is plausible that the observed increase of cellular GSH levels in rCalu- 6 cells was also due to the loss of the CBS inhibitor $\mathrm{UL} 3$ with consequent activation of the trans-sulfuration pathway via CBS that lead to increased availability of the GSH precursor cysteine.

All together, our results led us to propose a model by which upon acquired resistance, the activation of the defense response depends on the uL3 status (Figure 6). In particular, in the condition of multidrug resistance, the production of uL3 is down-regulated and the expression of stress-response genes is activated. Specifically, the binding of uL3 to $x C T$ and GST- $\alpha 1$ gene promoters decreases and, as a consequence, its inhibitory activity on these two promoters is suppressed. This represents an indirect way to potentiate the expression of antioxidant genes and activate the defense response. On the one hand, overexpression of the $\mathrm{xCT}$ light chain and the consequent increased activity of the antiporter system $\mathrm{xc}^{-}$and, on the other hand, the activation of the trans-sulfuration pathway via CBS induced by lower levels of uL3 [22], lead to increased availability of Cysteine, a GSH precursor crucial for cell stress response. In addition, uL3 down-regulation positively associated to the up-regulation of Pgp-expression, contributing to the efflux mediated resistance (Figure 6).

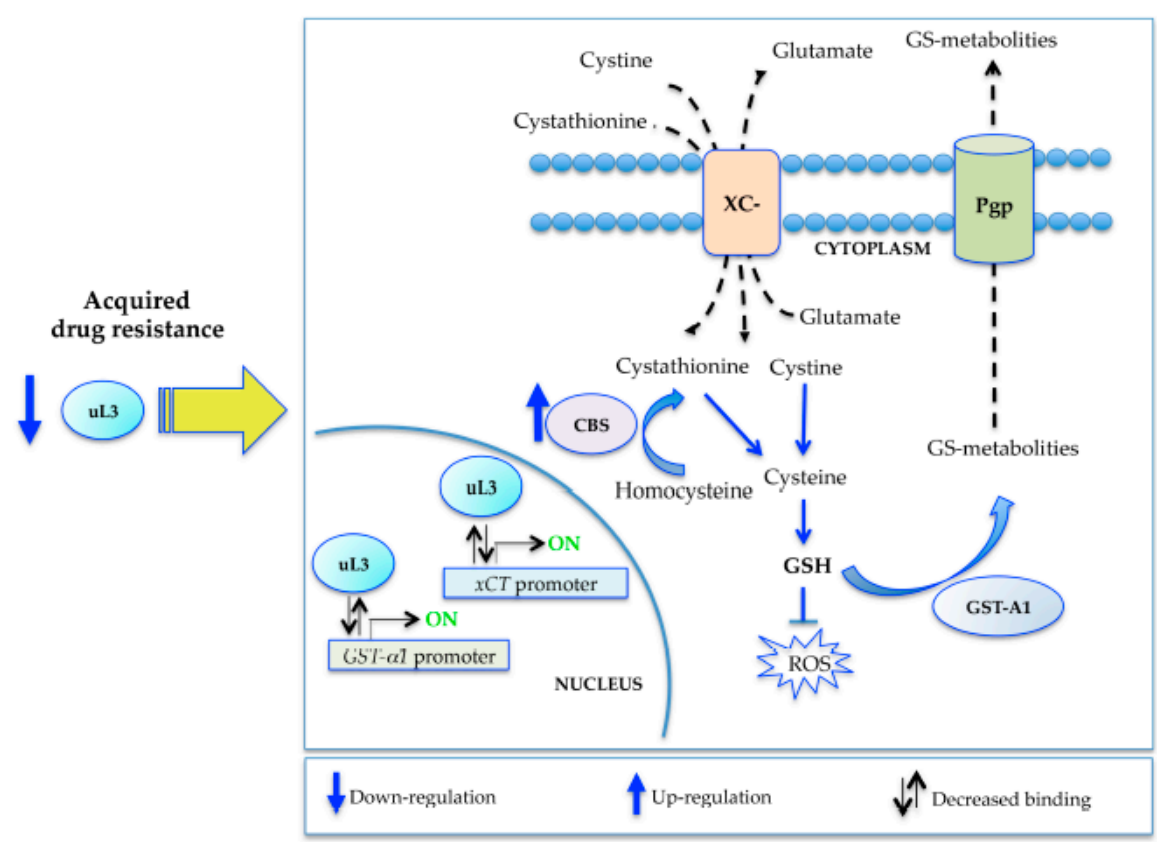

Figure 6. Proposed model of acquired multidrug resistance based on uL3 status.

\section{Materials and Methods}

\subsection{Cell Cultures, Drug Treatment, and Production of Multidrug Resistant Calu-6 Cells}

Human Calu-6 were cultured as previously described [43]. The uL3 $\Delta$ Calu- 6 cell line was obtained from Calu- 6 cells by transfecting $2 \mu \mathrm{g}$ of shRNA uL3 plasmid [21] by using Lipofectamin 2000 (Life Technologies, Carlsbad, CA, USA) according to the manufacturer's instructions.

The multidrug resistant Calu- 6 cell line (rCalu-6) was established from Calu- 6 cells by continuous exposure to increasing concentrations of 5-FU (from 2.5 to $25 \mu \mathrm{M}$ ). rCalu- 6 cells were then maintained in the presence of $20 \mu \mathrm{M} 5-\mathrm{FU}$.

For drug treatments, rCalu-6 cells were grown in medium without 5-FU for 2 weeks before 5-FU addition. 


\subsection{Cell Viability Assay and Clonogenic Assay}

The Sulforhodamine B (SRB) colorimetric assay for cell viability of Calu- 6 and rCalu- 6 cells and the clonogenic assay were performed as previously described [25].

\subsection{Glutathione Measurement}

Intracellular GSH concentration was measured using a commercially available kit (Chemicon, Temecula, CA, USA) according to the manufacturer's protocol.

\subsection{Glutamate Analysis}

Glutamate in the cell medium was assayed with Amplex Red assay kits (catalog number: A-12,221, Invitrogen, Carlsbad, CA, USA) according to the manufacturer's protocol. All treatments were performed in serum-free and L-glutamine-free MEM (Invitrogen).

\subsection{Cystine Uptake}

Cystine uptake was performed as previously described [44] by using $50 \mu \mathrm{m} \mathrm{L-[35S]-Cystine}$ (specific activity $40 \mathrm{mCi} / \mathrm{mmol}$ ).

\subsection{Reactive Oxygen Species (ROS) Assay}

Cells were seeded at a density of $1 \times 10^{4}$ in 96-well clear bottom black plates, allowed to equilibrate overnight, and were starved in serum free medium for $24 \mathrm{~h}$ followed by treatments. Intracelluar ROS levels were determined by using the Cell Meter ${ }^{\mathrm{TM}}$ Fluorimetric Intracellular Total ROS Activity Assay Kit*Red Fluorescence according to the manufacturer's guideline (catalog number: 22901, AAT Bioquest ${ }^{\circledR}$, Sunnyvale, CA, USA).

\subsection{DNA Constructs, siRNA, and Transfection}

DNA constructs containing $x$-CT and GST- $\alpha 1$ promoters, the plasmid encoding Flag-Nrf2, and the plasmid encoding uL3-HA (pHA-uL3) and eL8 (pHA-eL8) were already available [21]. siRNA against Nrf2 was purchased by Santa Cruz Biotechnology (catalog number: sc-37030, Santa Cruz, CA, USA). Transfections were performed as previously described [45].

\subsection{Dual Luciferase Assays}

The luciferase assay was performed with a Dual-Luciferase reporter assay system (Promega, Madison, WI, USA) according to the manufacturer's instructions. The firefly luciferase activity was normalized to Renilla internal control luminescence.

\subsection{Chromatin Immunoprecipitation}

The chromatin immunoprecipitation assay was performed as previously reported [19].

\subsection{RNA Isolation, RT, and Quantitative Real-Time PCR}

Total RNA from the cells was extracted as previously reported [46]. RT-qPCR was performed as previously reported [19]. The primers used for RT-qPCR were the following: TS (Forward: 5'-TTACCTGAATCACATCGAGC-3' ${ }^{\prime}$ and Reverse: $5^{\prime}$-ATATCCTTCGAGCTCCTTTG-3'); DPYD (Forward: 5'-GTTCTGGCTACCAGGCTAT-3' and Reverse: 5'-CATAAGGTGTTGTCCTGGAA-3'); $u$ L3 (Forward: 5'-CAAAGGCTACAAAGGGGT-3' ${ }^{\prime}$ and Reverse: $5^{\prime}$-CTCAGTGCGGTGATGGTAG-3'); MDR1 (Forward: 5'-ATGCCTTCATCGAGTCACTG-3' and Reverse: $5^{\prime}$-TAACAAGGGCACGAGCTATG-3'); Nrf2 (Forward: 5'-GAGAGCCCAGTCTTCATTGC-3' and Reverse: 5'-TGCTCAATGTCCTGTTGCAT-3); $x C T$ (Forward: $5^{\prime}$-AAACTTGCTAAGCTCTGTGTTGG-3' ${ }^{\prime}$, Reverse: $5^{\prime}$-CACATCACATGTTTGTACA CTCG-3'); GST- $\alpha 1$ (Forward: 5'-CAGCAAGTGCCAATGGTTGA-3' and Reverse: 5'-TATTTGCTG 
GCAATGTAGTTGAGAA-3'); $\beta$-actin (Forward: 5' GGCGGCACCACCATGTACCCT-3' and Reverse: $5^{\prime}$-AGGGGCCGGACTCGTCATACT-3'). The primers used for $C B S$ were previously reported [22].

The association of $\mathrm{uL} 3$ with endogenous $x$-CT and GST- $\alpha 1$ promoters was measured by qPCR on immunoprecipitated chromatin as previously described [47].

The PCR products were run on agarose gels and visualized by ethidium bromide staining.

\subsection{Western Blot Analysis}

Western blotting analysis was performed as previously reported [48]. The membranes were incubated with anti-uL3 and anti-eL8 (Primm, Milan, Italy), anti-xCT and anti-GST-A1, anti-TS, anti-DPYD and anti-Pgp (Abcam, Cambridge, MA, USA), anti-eS19 (Sigma-Aldrich, St Louis, MO, USA), anti-CBS, and anti- $\beta$-actin (Santa Cruz Biotechnology). Proteins were visualized with enhanced chemiluminescence [49]. Densitometric analysis was carried out using ImageJ software (Image 1.49v, available on: http:/ /imagej.nih.gov/ij, National Institutes of Health, Bethesda, MD, USA).

\subsection{Immunoprecipitation Assay}

The immunoprecipitation assay was performed as previously described [50] by using anti-Flag (Santa Cruz Biotechnology). The beads were washed and boiled in the SDS sample buffer. The eluted proteins were loaded on $12 \%$ SDS-PAGE and analyzed by Western blotting.

\subsection{Statistical Analysis}

Statistical analysis was performed as previously reported [51]. Statistical comparisons were made by Student's $t$-test and one-way ANOVA. $p<0.05$ was considered significant, $p<0.01$ was considered highly significant.

\section{Conclusions}

In conclusion, in this study we provide new insights concerning a rational applicability of the combined treatment based on 5-FU plus uL3 [24] as an individualized therapy for tumors lacking uL3, in an attempt to overcome drug resistance and yield better clinical outcomes.

Supplementary Materials: Supplementary materials can be found at www.mdpi.com/1422-0067/18/3/547/s1.

Acknowledgments: This work was supported by the Ministero della Università e della Ricerca (MIUR) PRIN 2010-2011, (4 AE23N_006) to Giulia Russo, Regione Campania 2007 (L.R. N.5 del 28.03.2002), Regione Campania 2005 (L.R. N.5 del 28.03.2002, prot. A.10057.RusGRC05C) to Giulia Russo.

Author Contributions: Annapina Russo and Giulia Russo conceived and designed the experiments; Annapina Russo, Assunta Saide, Silvia Smaldone performed the experiments; Annapina Russo, Giulia Russo, and Raffaella Faraonio analyzed the data; Annapina Russo and Giulia Russo wrote the paper. All authors read and approved the final manuscript.

Conflicts of Interest: The authors declare no conflict of interest.

\section{References}

1. DeSantis, C.E.; Lin, C.C.; Mariotto, A.B.; Siegel, R.L.; Stein, K.D.; Kramer, J.L.; Alteri, R.; Robbins, A.S.; Jemal, A. Cancer treatment and survivorship statistics, 2014. CA Cancer J. Clin. 2014, 64, 252-271. [CrossRef] [PubMed]

2. Maione, P.; Perrone, F.; Gallo, C.; Manzione, L.; Piantedosi, F.; Barbera, S.; Cigolari, S.; Rosetti, F.; Piazza, E.; Robbiati, S.F.; et al. Pretreatment quality of life and functional status assessment significantly predict survival of elderly patients with advanced non-small-cell lung cancer receiving chemotherapy: A prognostic analysis of the multicenter italian lung cancer in the elderly study. J. Clin. Oncol. 2005, 23, 6865-6872. [PubMed]

3. Chang, A. Chemotherapy, chemoresistance and the changing treatment landscape for NSCLC. Lung Cancer 2011, 71, 3-10. [CrossRef] [PubMed]

4. Chunga, Y.M.; Parka, S.; Parka, J.K.; Kimb, Y.; Kanga, Y.; Yooa, Y.D. Establishment and characterization of 5-fuorouracil-resistant gastric cancer cells. Cancer Lett. 2000, 159, 95-101. [CrossRef] 
5. Gu, W.; Fang, F.-F.; Li, B.; Cheng, B.-B.; Ling, C.-Q. Characterization and resistance mechanisms of a 5-fluorouracil-resistant hepatocellular carcinoma cell line. Asian Pac. J. Cancer Prev. 2012, 13, 4807-4814. [CrossRef] [PubMed]

6. Longley, D.B.; Harkin, D.P.; Johnston, P.G. 5-Fluorouracil: Mechanisms of action and clinical strategies. Nat. Rev. Cancer 2003, 3, 330-338. [CrossRef] [PubMed]

7. Boulon, S.; Westman, B.J.; Hutten, S.; Boisvert, F.M.; Lamond, A.I. The nucleolus under stress. Mol. Cell 2010, 40, 216-227. [CrossRef] [PubMed]

8. Lewinska, A.; Wnuk, M.; Grzelak, A.; Bartosz, G. Nucleolus as an oxidative stress sensor in the yeast saccharomyces cerevisiae. Redox Rep. 2010, 15, 87-96. [CrossRef] [PubMed]

9. Challagundla, K.B.; Sun, X.X.; Zhang, X.; de Vine, T.; Zhang, Q.; Sears, R.C.; Dai, M.S. Ribosomal protein L11 recruits miR-24/miRISC to repress c-Myc expression in response to ribosomal stress. Mol. Cell. Biol. 2011, 31, 4007-4021. [CrossRef] [PubMed]

10. De Marval, P.L.M.; Zhang, Y. The RP-Mdm2-p53 pathway and tumorigenesis. Oncotarget 2011, $234-238$. [CrossRef] [PubMed]

11. Cuccurese, M.; Russo, G.; Russo, A.; Pietropaolo, C. Alternative splicing and nonsense-mediated mRNA decay regulate mammalian ribosomal gene expression. Nucleic Acids Res. 2005, 33, 5965-5977. [CrossRef] [PubMed]

12. Russo, G.; Cuccurese, M.; Monti, G.; Russo, A.; Amoresano, A.; Pucci, P.; Pietropaolo, C. Ribosomal protein L7a binds RNA through two distinct RNA-binding domains. Biochem. J. 2005, 385, 289-299. [CrossRef] [PubMed]

13. Russo, A.; Russo, G.; Cuccurese, M.; Garbi, C.; Pietropaolo, C. The 3'-untranslated region directs ribosomal protein-encoding mRNAs to specific cytoplasmic regions. Biochim. Biophys. Acta 2006, 1763, 833-843. [CrossRef] [PubMed]

14. Russo, A.; Cirulli, C.; Amoresano, A.; Pucci, P.; Pietropaolo, C.; Russo, G. Cis-acting sequences and trans-acting factors in the localization of mRNA for mitochondrial ribosomal proteins. Biochim. Biophys. Acta 2008, 1779, 820-829. [CrossRef] [PubMed]

15. Russo, A.; Russo, G. Ribosomal proteins control or bypass p53 during nucleolar stress. Int. J. Mol. Sci. 2017, 18. [CrossRef] [PubMed]

16. Ban, N.; Beckmann, R.; Cate, J.H.; Dinman, J.D.; Dragon, F.; Ellis, S.R.; Lafontaine, D.L.; Lindahl, L.; Liljas, A.; Lipton, J.M.; et al. A new system for naming ribosomal proteins. Curr. Opin. Struct. Biol. 2014, 24, 165-169. [CrossRef] [PubMed]

17. Russo, A.; Siciliano, G.; Catillo, M.; Giangrande, C.; Amoresano, A.; Pucci, P.; Pietropaolo, C.; Russo, G. hnRNP H1 and intronic G runs in the splicing control of the human rpL3 gene. Biochim. Biophys. Acta 2010, 1799, 419-428. [CrossRef] [PubMed]

18. Russo, A.; Catillo, M.; Esposito, D.; Briata, P.; Pietropaolo, C.; Russo, G. Autoregulatory circuit of human rpL3 expression requires hnRNP H1, NPM and KHSRP. Nucleic Acids Res. 2011, 39, 7576-7585. [CrossRef] [PubMed]

19. Russo, A.; Esposito, D.; Catillo, M.; Pietropaolo, C.; Crescenzi, E.; Russo, G. Human rpL3 induces G $1 / \mathrm{S}$ arrest or apoptosis by modulating p21 waf1/cip1 levels in a p53-independent manner. Cell Cycle 2013, 12, 76-87. [CrossRef] [PubMed]

20. Esposito, D.; Crescenzi, E.; Sagar, V.; Loreni, F.; Russo, A.; Russo, G. Human rpL3 plays a crucial role in cell response to nucleolar stress induced by 5-FU and L-OHP. Oncotarget 2014, 5, 11737-11751. [CrossRef] [PubMed]

21. Russo, A.; Pagliara, V.; Albano, F.; Esposito, D.; Sagar, V.; Loreni, F.; Irace, C.; Santamaria, R.; Russo, G. Regulatory role of rpL3 in cell response to nucleolar stress induced by act D in tumor cells lacking functional p53. Cell Cycle 2016, 15, 41-51. [CrossRef] [PubMed]

22. Russo, A.; Saide, A.; Cagliani, R.; Cantile, M.; Botti, G.; Russo, G. rpL3 promotes the apoptosis of p53 mutated lung cancer cells by down-regulating CBS and NFkB upon 5-FU treatment. Sci. Rep. 2016, 6, 38369. [CrossRef] [PubMed]

23. Pagliara, V.; Saide, A.; Mitidieri, E.; di Villa Bianca, D.R.; Sorrentino, R.; Russo, G.; Russo, A. 5-Fu targets rpL3 to induce mitochondrial apoptosis via cystathionine- $\beta$-synthase in colon cancer cells lacking p53. Oncotarget 2016, 7, 50333-50348. [CrossRef] [PubMed] 
24. Russo, A.; Maiolino, S.; Pagliara, V.; Ungaro, F.; Tatangelo, F.; Leone, A.; Scalia, G.; Budillon, A.; Quaglia, F.; Russo, G. Enhancement of 5-FU sensitivity by the proapoptotic rpL3 gene in p53 null colon cancer cells through combined polymer nanoparticles. Oncotarget 2016, 7, 79670-79687. [CrossRef] [PubMed]

25. Russo, A.; Pellosi, D.S.; Pagliara, V.; Milone, M.R.; Pucci, B.; Caetano, W.; Hioka, N.; Budillon, A.; Ungaro, F.; Russo, G.; et al. Biotin-targeted pluronic ${ }^{\circledR}$ P123/F127 mixed micelles delivering niclosamide: A repositioning strategy to treat drug-resistant lung cancer cells. Int. J. Pharm. 2016, 511, 127-139. [CrossRef] [PubMed]

26. Shen, D.W.; Liang, X.J.; Suzuki, T.; Gottesman, M.M. Identification by functional cloning from a retroviral cDNA library of cDNAs for ribosomal protein 136 and the 10-kDa heat shock protein that confer cisplatin resistance. Mol. Pharmacol. 2006, 69, 1383-1388. [CrossRef] [PubMed]

27. Yang, H.J.; Youn, H.; Seong, K.M.; Jin, Y.W.; Kim, J.; Youn, B. Phosphorylation of ribosomal protein S3 and antiapoptotic TRAF2 protein mediates radioresistance in non-small cell lung cancer cells. J. Biol. Chem. 2013, 288, 2965-2975. [CrossRef] [PubMed]

28. Guo, Y.-L.; Kong, Q.-S.; Liu, H.-S.; Tan, W.-B. Drug resistance effects of ribosomal protein L24 overexpression in hepatocellular carcinoma HepG2 cells. Asian Pac. J. Cancer Prev. 2014, 15, 9853-9857. [CrossRef] [PubMed]

29. Baguley, B.C. Multiple drug resistance mechanisms in cancer. Mol. Biotechnol. 2010, 46, 308-316. [CrossRef] [PubMed]

30. Takahashi, K.; Tanaka, M.; Inagaki, A.; Wanibuchi, H.; Izumi, Y.; Miura, K.; Nagayama, K.; Shiota, M.; Iwao, H. Establishment of a 5-fluorouracil-resistant triple-negative breast cancer cell line. Int. J. Oncol. 2013, 43, 1985-1991. [PubMed]

31. Hayes, J.D.; McLellan, L.I. Glutathione and glutathione-dependent enzymes represent a co-ordinately regulated defence against oxidative stress. Free Radic. Res. 1999, 31, 273-300. [CrossRef] [PubMed]

32. Traverso, N.; Ricciarelli, R.; Nitti, M.; Marengo, B.; Furfaro, A.L.; Pronzato, M.A.; Marinari, U.M.; Domenicotti, C. Role of glutathione in cancer progression and chemoresistance. Oxid. Med. Cell. Longev. 2013, 2013, 972913. [CrossRef] [PubMed]

33. Homma, S.; Ishii, Y.; Morishima, Y.; Yamadori, T.; Matsuno, Y.; Haraguchi, N.; Kikuchi, N.; Satoh, H.; Sakamoto, T.; Hizawa, N.; et al. Nrf2 enhances cell proliferation and resistance to anticancer drugs in human lung cancer. Clin. Cancer Res. 2009, 15, 3423-3432. [CrossRef] [PubMed]

34. Faraonio, R.; Vergara, P.; di Marzo, D.; Napolitano, M.; Russo, T.; Cimino, F. Transcription regulation in NIH3T3 cell clones resistant to diethylmaleate-induced oxidative stress and apoptosis. Antioxid. Redox Signal. 2006, 8, 365-374. [CrossRef] [PubMed]

35. Sparaneo, A.; Fabrizio, F.P.; Muscarella, L.A. Nrf2 and notch signaling in lung cancer: Near the crossroad. Oxid. Med. Cell. Longev. 2016, 2016, 7316492. [CrossRef] [PubMed]

36. Sharma, M.K.; Seidlitz, E.P.; Singh, G. Cancer cells release glutamate via the cystine/glutamate antiporter. Biochem. Biophys. Res. Commun. 2010, 391, 91-95. [CrossRef] [PubMed]

37. Miniaci, M.C.; Irace, C.; Capuozzo, A.; Piccolo, M.; di Pascale, A.; Russo, A.; Lippiello, P.; Lepre, F.; Russo, G.; Santamaria, R. Cysteine prevents the reduction in keratin synthesis induced by iron deficiency in human keratinocytes. J. Cell. Biochem. 2016, 117, 402-412. [CrossRef] [PubMed]

38. Maiolino, S.; Russo, A.; Pagliara, V.; Conte, C.; Ungaro, F.; Russo, G.; Quaglia, F. Biodegradable nanoparticles sequentially decorated with polyethyleneimine and hyaluronan for the targeted delivery of docetaxel to airway cancer cells. J. Nanobiotechnol. 2015, 13, 29. [CrossRef] [PubMed]

39. Kobayashi, S.; Sato, M.; Kasakoshi, T.; Tsutsui, T.; Sugimoto, M.; Osaki, M.; Okada, F.; Igarashi, K.; Hiratake, J.; Homma, T.; et al. Cystathionine is a novel substrate of cystine/glutamate transporter: Implications for immune function. J. Biol. Chem. 2015, 290, 8778-8788. [CrossRef] [PubMed]

40. Di Villa Bianca, D.R.; Mitidieri, E.; Esposito, D.; Donnarumma, E.; Russo, A.; Fusco, F.; Ianaro, A.; Mirone, V.; Cirino, G.; Russo, G.; et al. Human cystathionine- $\beta$-synthase phosphorylation on serine 227 modulates hydrogen sulfide production in human urothelium. PLoS ONE 2015, 10, e0136859. [CrossRef] [PubMed]

41. Di Villa Bianca, D.R.; Mitidieri, E.; Fusco, F.; Russo, A.; Pagliara, V.; Tramontano, T.; Donnarumma, E.; Mirone, V.; Cirino, G.; Russo, G.; et al. Urothelium muscarinic activation phosphorylates CBS ${ }^{\mathrm{Se} 227}$ via cGMP/PKG pathway causing human bladder relaxation through $\mathrm{H}_{2} \mathrm{~S}$ production. Sci. Rep. 2016, 6, 31491. [CrossRef] [PubMed]

42. Bhattacharyya, S.; Saha, S.; Giri, K.; Lanza, I.R.; Nair, K.S.; Jennings, N.B.; Rodriguez-Aguayo, C.; Lopez-Berestein, G.; Basal, E.; Weaver, A.L.; et al. Cystathionine $\beta$-synthase (CBS) contributes to advanced ovarian cancer progression and drug resistance. PLoS ONE 2013, 8, e79167. [CrossRef] [PubMed] 
43. Esposito, V.; Russo, A.; Amato, T.; Varra, M.; Vellecco, V.; Bucci, M.; Russo, G.; Virgilio, A.; Galeone, A. Backbone modified TBA analogues endowed with antiproliferative activity. Biochim. Biophys. Acta 2016. [CrossRef] [PubMed]

44. Murphy, T.H.; Miyamoto, M.; Sastre, A.; Schnaar, R.L.; Coyle, J.T. Glutamate toxicity in a neuronal cell line involves inhibition of cystine transport leading to oxidative stress. Cell Press 1989, 2, 1547-1558. [CrossRef]

45. Lavecchia, A.; di Giovanni, C.; Cerchia, C.; Russo, A.; Russo, G.; Novellino, E. Discovery of a novel small molecule inhibitor targeting the frataxin/ubiquitin interaction via structure-based virtual screening and bioassays. J. Med. Chem. 2013, 56, 2861-2873. [CrossRef] [PubMed]

46. De Filippis, D.; Russo, A.; D’Amico, A.; Esposito, G.; Pietropaolo, C.; Cinelli, M.; Russo, G.; Iuvone, T. Cannabinoids reduce granuloma-associated angiogenesis in rats by controlling transcription and expression of mast cell protease-5. Br. J. Pharmacol. 2008, 154, 1672-1679. [CrossRef] [PubMed]

47. Faraonio, R.; Vergara, P.; di Marzo, D.; Pierantoni, M.G.; Napolitano, M.; Russo, T.; Cimino, F. p53 suppresses the Nrf2-dependent transcription of antioxidant response genes. J. Biol. Chem. 2006, 281, 39776-39784. [CrossRef] [PubMed]

48. Russo, A.; Russo, G.; Peticca, M.; Pietropaolo, C.; di Rosa, M.; Iuvone, T. Inhibition of granuloma-associated angiogenesis by controlling mast cell mediator release: Role of mast cell protease-5. Br. J. Pharmacol. 2005, 145, 24-33. [CrossRef] [PubMed]

49. De Nigris, F.; Mancini, F.P.; Schiano, C.; Infante, T.; Zullo, A.; Minucci, P.B.; Al-Omran, M.; Giordano, A.; Napoli, C. Osteosarcoma cells induce endothelial cell proliferation during neo-angiogenesis. J. Cell. Physiol. 2013, 228, 846-852. [CrossRef] [PubMed]

50. De Filippis, D.; Russo, A.; de Stefano, D.; Cipriano, M.; Esposito, D.; Grassia, G.; Carnuccio, R.; Russo, G.; Iuvone, T. Palmitoylethanolamide inhibits $\mathrm{rMCP}-5$ expression by regulating MITF activation in rat chronic granulomatous inflammation. Eur. J. Pharmacol. 2014, 725, 64-69. [CrossRef] [PubMed]

51. De Filippis, D.; Russo, A.; de Stefano, D.; Maiuri, M.C.; Esposito, G.; Cinelli, M.P.; Pietropaolo, C.; Carnuccio, R.; Russo, G.; Iuvone, T. Local administration of win 55,21-2 reduces chronic granuloma-associated angiogenesis in rat by inhibiting NF-кB activation. J. Mol. Med. 2007, 85, 635-645. [CrossRef] [PubMed]

(C) 2017 by the authors. Licensee MDPI, Basel, Switzerland. This article is an open access article distributed under the terms and conditions of the Creative Commons Attribution (CC BY) license (http:/ / creativecommons.org/licenses/by/4.0/). 